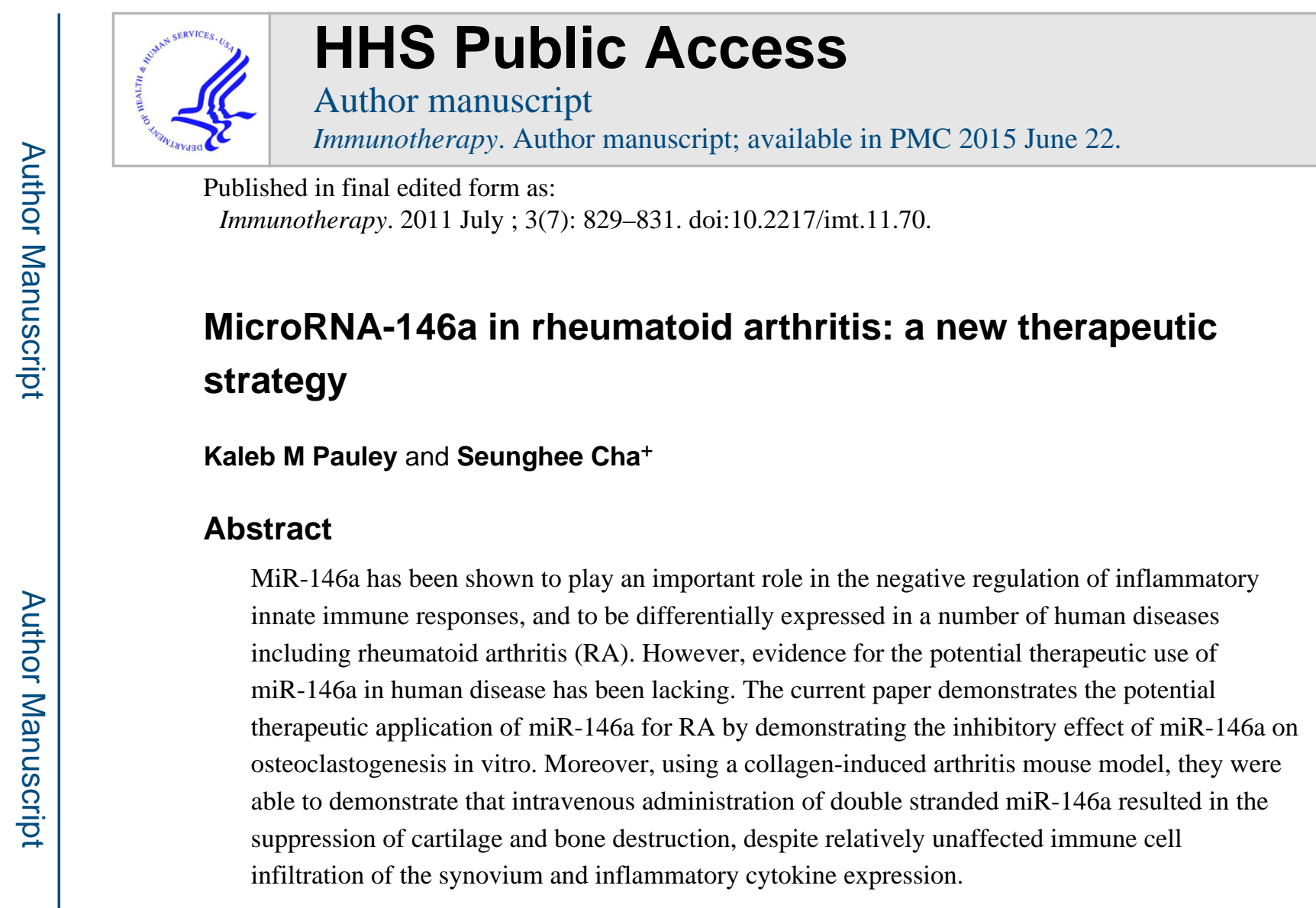

\title{
Keywords
}

microRNA-146a; rheumatoid arthritis; microRNA therapy; autoimmune disease;

osteoclastogenesis

MicroRNAs (miRNAs) are small non-coding regulatory RNA molecules that function by binding to the 3'UTR of their target mRNA(s) and leading to degradation or translational repression [2]. MiRNAs are now known to play important regulatory roles in a variety of cellular processes including development, cell cycle, and apoptosis. In the immune system, certain miRNAs have emerged as key regulators of immune cell development and innate/ adaptive immune responses [3].

In 2006, miR-146a was found to be one of several miRNAs that demonstrated increased expression in response to LPS and other inflammatory stimuli in the human monocytic cell line THP-1 [4]. Furthermore, it was demonstrated that miR-146a targets TNF receptorassociated factor 6 (TRAF6) and IL-1 receptor-associated kinase 1 (IRAK1), two key adaptor molecules in the TLR and IL-1 receptor signaling pathways $[4,5]$. Since then, miR-146a has been shown to suppress NF- $\kappa$ B activity [5], suppress the LPS-induced inflammatory response [6-9], and play a role in the development of endotoxin tolerance [10, 11]. Overall, it is clear that miR-146a plays a critical role in regulating inflammatory responses through a negative feedback pathway.

${ }^{+}$Author for correspondence: University of Florida College of Dentistry, Department of Oral and Maxillofacial Diagnostic Sciences, 1600 SW Archer Rd., Gainesville, FL 32610 USA, Tel.: +1 352273 6687, Fax: +1 352846 0588, scha@ dental.ufl.edu. 
Given this role, it is not surprising that miR-146a has been shown to be differentially expressed in a number of inflammatory autoimmune diseases including systemic lupus erythematosus (SLE) [12-15], Sjögren's syndrome (SjS) [8], and rheumatoid arthritis (RA) [16-18]. Specifically, it has been reported that miR-146a is highly expressed in synovial tissue and synovial fibroblasts of RA patients compared to normal individuals and osteoarthritis patients $[16,18]$. It has also been demonstrated that miR-146a is upregulated in the PBMCs of RA patients compared to healthy controls, and that this upregulation correlates with disease activity [17]. Despite these findings, the role of miR-146a in RA pathogenesis or its potential as a therapeutic target or diagnostic marker have not been explored until the current paper.

\section{Summary of methods and results}

In the current paper, Nakasa et al. demonstrate the potential use of double stranded (ds) miR-146a as a new therapeutic strategy in RA. They transfected ds miR-146a or nonspecific dsRNA into the $\mathrm{CD} 14^{+}$cell population of human PBMCs and then induced osteoclastogenesis by treatment with M-CSF and TNFa or RANKL for three weeks. Three days post-transfection, TRAF6 protein levels were monitored by immunofluorescence and found to be significantly reduced in ds miR-146a transfected cells. The number of osteoclasts (defined as TRAP-positive cells with more than three nuclei) in cells transfected with ds miR-146a was significantly reduced in a dose dependent manner compared to cells transfected with nonspecific dsRNA. Osteoclast function was examined by a bone resorption assay and was found to be significantly inhibited as evidenced by the lack of pit formation by ds miR-146a-transfected cells. The expression of representative osteoclast marker genes c-Jun, NFATc1, PU.1, and TRAP was also examined by quantitative RT-PCR three days after transfection and found to be significantly downregulated after transfection with ds miR-146a compared to non-specific dsRNA. This inhibition of osteoclast formation was observed in both osteoclastogenesis culture systems (M-CSF+TNFa or M-CSF+RANKL).

To test the efficacy of ds miR-146a inhibition of bone destruction in vivo, ds miR-146a was administered intravenously to arthritic mice. Collagen-induced arthritis (CIA) was induced in 8 week old DBA/1 mice by injecting collagen emulsified in complete Freund's adjuvant

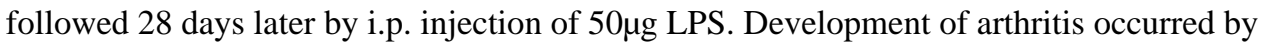
day 30 and was monitored in each limb by a scoring system of 0 to 4 with $0=$ no inflammation and $4=$ maximally inflamed limb with involvement of multiple joints. Starting on day 30, ds miR-146a or non-specific dsRNA was i.v. injected twice at 1-week intervals.

Four weeks after the first injection, the extent of bone destruction was reduced in mice injected with ds miR-146a compared to non-specific dsRNA. Additionally, histological analysis showed extensive pannus formation with synovial hyperplasia, a large number of inflammatory cell infiltrate, and cartilage and bone destruction in the hind paw of mice treated with non-specific dsRNA. In comparison, there was no bone or cartilage destruction detected in the hind paw of mice treated with ds miR-146a, but synovial hyperplasia was not completely inhibited. TRAP-positive cells were detected at the border of the pannus and bone in the non-specific dsRNA treated group, while few or none were observed in the ds miR-146a treated group. Despite the inhibition of bone/cartilage destruction by ds miR-146a 
injection, there was no significant difference in the arthritis score between the ds miR-146a treated group and the non-specific dsRNA treated group. TNFa, IL- $1 \beta$, and IL- 6 expression were also analyzed in the synovium by immunofluorescence and found to be downregulated in ds miR-146a treated mice compared to the non-specific dsRNA treated group. However, injection of ds miR-146a was not able to completely suppress inflammatory cytokine expression.

\section{Discussion}

As the physiological roles of miRNAs are better characterized and understood, the potential for using or targeting miRNAs as a therapeutic strategy in disease increases. miR-146a has been shown to play important roles in the regulation of inflammatory immune responses [19], and has been shown to be overexpressed in RA [16-18] and $\mathrm{SjS}$ [8], and underexpressed in SLE [12-15] making it an interesting target for diagnostic purposes and therapeutic use in these rheumatic diseases. The current paper outlines a new function for miR-146a in inhibiting osteoclastogenesis. The mechanism of this inhibition is unknown, but hypothesized to act through negative regulation of $\mathrm{NF \kappa B}$ activity in the TNFa-induced culture system, and negative regulation of TRAF6 in the RANKL-induced culture system. This raises an interesting question of why, if miR-146a is already overexpressed in RA patients, can it not inhibit osteoclastogenesis and bone destruction in these patients. Further studies are needed to investigate why overexpressed miR-146a in $\mathrm{RA}$ and $\mathrm{SjS}$ is unable to effectively downregulate the chronic inflammatory conditions in these diseases, and if administration of ds miR-146a would be effective in these situations. Nonetheless, the current study is the first to attempt the therapeutic use of miRNA for an autoimmune disease.

\section{Future perspective}

The current paper by Nakasa et al. demonstrates that administration of ds miR-146a in arthritic mice was capable of preventing bone/cartilage destruction, but unable to completely ameliorate joint inflammation. These data suggest that administration of ds miR-146a alone may not be a sufficient treatment for RA patients and perhaps ds miR-146a in combination with other biological agents or with other miRNAs would be more effective at ameliorating the chronic inflammatory response. Additionally, further investigation is needed to identify the localization of ds miR-146a uptake after i.v. administration and to optimize application regimens such as dose or delivery routes. The use of ds miRNAs as therapy has potential applications in a number of diseases including cancers, cardiovascular disease, and autoimmune diseases; however, numerous challenges still remain in designing effective and safe delivery of these agents in a cell or tissue-specific manner.

\section{Bibliography}

1. Nakasa T, Shibuya H, Nagata Y, Niimoto T, Ochi M. The inhibitory effect of microRNA-146 expression on bone destruction in arthritis. Arthritis Rheum. 2011

2. Filipowicz W, Bhattacharyya SN, Sonenberg N. Mechanisms of post-transcriptional regulation by microRNAs: are the answers in sight? Nat Rev Genet. 2008; 9(2):102-114. [PubMed: 18197166] 
3. Pauley KM, Cha S, Chan EK. MicroRNA in autoimmunity and autoimmune diseases. J Autoimmun. 2009; 32(3-4):189-194. [PubMed: 19303254]

4. Taganov KD, Boldin MP, Chang KJ, Baltimore D. NF-B-dependent induction of microRNA miR-146, an inhibitor targeted to signaling proteins of innate immune responses. Proc Natl Acad Sci U S A. 2006; 103:12481-12486. [PubMed: 16885212]

5. Bhaumik D, Scott GK, Schokrpur S, et al. MicroRNAs miR-146a/b negatively modulate the senescence-associated inflammatory mediators IL-6 and IL-8. Aging (Albany NY). 2009; 1(4):402411. [PubMed: 20148189]

6. Hou J, Wang P, Lin L, et al. MicroRNA-146a feedback inhibits RIG-I-dependent Type I IFN production in macrophages by targeting TRAF6, IRAK1, and IRAK2. J Immunol. 2009; 183(3): 2150-2158. [PubMed: 19596990]

7. Pauley KM, Satoh M, Pauley BA, et al. Formation of GW/P bodies as marker for microRNAmediated regulation of innate immune signaling in THP-1 cells. Immunol Cell Biol. 2009; 88(2): 205-212. [PubMed: 19918258]

8. Pauley KM, Stewart CM, Gauna AE, et al. Altered miR-146a expression in Sjogren's syndrome and its functional role in innate immunity. Eur J Immunol. 2011

9. Perry MM, Moschos SA, Williams AE, Shepherd NJ, Larner-Svensson HM, Lindsay MA. Rapid changes in microRNA-146a expression negatively regulate the IL-1beta-induced inflammatory response in human lung alveolar epithelial cells. J Immunol. 2008; 180(8):5689-5698. [PubMed: 18390754]

10. Nahid MA, Pauley KM, Satoh M, Chan EK. miR-146a is critical for endotoxin-induced tolerance: IMPLICATION IN INNATE IMMUNITY. J Biol Chem. 2009; 284(50):34590-34599. [PubMed: 19840932]

11. Nahid MA, Satoh M, Chan EK. Mechanistic role of microRNA-146a in endotoxin-induced differential cross-regulation of TLR signaling. J Immunol. 2011; 186(3):1723-1734. [PubMed: 21178010]

12. Dai Y, Huang YS, Tang M, et al. Microarray analysis of microRNA expression in peripheral blood cells of systemic lupus erythematosus patients. Lupus. 2007; 16(12):939-946. [PubMed: 18042587]

13. Dai Y, Sui W, Lan H, Yan Q, Huang H, Huang Y. Comprehensive analysis of microRNA expression patterns in renal biopsies of lupus nephritis patients. Rheumatol Int. 2008; 29(7):749754. [PubMed: 18998140]

14. Tang Y, Luo X, Cui H, et al. MicroRNA-146A contributes to abnormal activation of the type I interferon pathway in human lupus by targeting the key signaling proteins. Arthritis Rheum. 2009; 60(4):1065-1075. [PubMed: 19333922]

15. Wang G, Tam LS, Li EK, et al. Serum and urinary cell-free MiR-146a and MiR-155 in patients with systemic lupus erythematosus. J Rheumatol. 2010; 37(12):2516-2522. [PubMed: 20952466]

16. Nakasa T, Miyaki S, Okubo A, et al. Expression of microRNA-146 in rheumatoid arthritis synovial tissue. Arthritis Rheum. 2008; 58(5):1284-1292. [PubMed: 18438844]

17. Pauley KM, Satoh M, Chan AL, Bubb MR, Reeves WH, Chan EK. Upregulated miR-146a expression in peripheral blood mononuclear cells from rheumatoid arthritis patients. Arthritis Res Ther. 2008; 10(4):R101. [PubMed: 18759964]

18. Stanczyk J, Pedrioli DM, Brentano F, et al. Altered expression of MicroRNA in synovial fibroblasts and synovial tissue in rheumatoid arthritis. Arthritis Rheum. 2008; 58(4):1001-1009. [PubMed: 18383392]

19. Li L, Chen XP, Li YJ. MicroRNA-146a and human disease. Scand J Immunol. 2010; 71(4):227231. [PubMed: 20384865] 


\section{Executive summary}

- MiR-146a has been shown to be overexpressed in the synovial tissue, synovial fibroblasts, and PBMCs of RA patients compared to controls and is known to play a role in downregulating inflammatory immune responses.

- The paper under evaluation demonstrated that miR-146a inhibits osteoclast development and that injection of ds miR-146a into arthritic mice resulted in the prevention of bone and cartilage destruction and suppression, but not complete inhibition of joint inflammation.

- These results are promising for the development of miRNA-based therapeutic strategies for autoimmune diseases. 